\title{
Genetic association of G2/M checkpoint genes with susceptibility to HIV-1 infection and AIDS progression in northern Chinese MSM population
}

Jiawei Wu

Harbin Medical University

\section{Xuelong Zhang}

Harbin Medical University

\section{Kaili Wang}

Harbin Medical University

Lidan Xu

Harbin Medical University

\section{Bangquan Liu}

Harbin Medical University

\section{Wenjing Sun}

Harbin Medical University

Yuanting Hu

Harbin Medical University

\section{Xueyuan Jia}

Harbin Medical University

\section{Haiming Sun}

Harbin Medical University

Jie Wu

Harbin Medical University

\section{Yun Huang}

Harbin Medical University

Wei Ji

Harbin Medical University

\section{Komal Saleem}

Harbin Medical University

\section{Songbin Fu}

Harbin Medical University

Yuandong Qiao ( $\nabla$ qiaoyuandong@hrbmu.edu.cn )

Harbin Medical University

\section{Research}

Keywords: G2/M checkpoint, SNP, MSM population, HIV-1, AIDS 
DOI: https://doi.org/10.21203/rs.3.rs-57482/v1

License: (c) (i) This work is licensed under a Creative Commons Attribution 4.0 International License. Read Full License 


\section{Abstract \\ Background}

MSM has a high risk of HIV infection. Previous studies showed that the cell cycle regulation played an important role in HIV-1 infection, especially in G2/M checkpoint. The ATR, Chk1, Cdc25C and CDK1 are key genes in G2/M checkpoint. However, the association between the SNPs of these genes and susceptibility to HIV-1 infection and AIDS progression remains unknown.

\section{Results}

42 tSNPs of G2/M checkpoint genes were genotyped to analyze the association between these tSNPs and susceptibility to HIV-1 infection and AIDS progression among MSM (529 HIV - 1 seropositive men and 529 HIV-1 seronegative men) in northern China. The results showed that rs34660854-A and rs75368165-A in ATR gene and rs3756766-A in Cdc25C gene could increase the risk of HIV- 1 infection $(P=0.049, P=0.020$ and $P=0.010$, respectively), and ATR rs75069062 and Chk1 rs10893405 were significantly associated with AIDS progression ( $P=$ 0.026 and $P=0.029$, respectively). Besides, rs34660854 and rs75368165 in ATR gene, rs12576279 and rs540436 in Chk1 gene, rs3756766 in Cdc25C gene and rs139245206 in CDK1 gene were significantly associated with HIV-1 infection under different models $(P<0.05)$.

\section{Conclusions}

The genetic variants of G2/M checkpoint genes had a molecular impact on the genesis of HIV- 1 infection and AIDS progression in northern Chinese MSM population.

\section{Background}

Acquired immune deficiency syndrome (AIDS) is an autoimmune disease caused by Human immunodeficiency virus (HIV), a typical retrovirus. Up till now, it is still unable to cure and seriously endangers global public health security. In recent years, the number of HIV-1 infected people has progressively increased and the proportion of MSM is increasing year by year.[1]

The cell cycle is a complex process that is involved in many important physiological processes such as the cell proliferation and diseases pathogenesis. $[2,3]$ Cell cycle checkpoints regulate the transition from one phase to another in turn to ensure the accuracy of cell cycle.[4] The G2/M checkpoint is responsible for G2 phase to M phase after DNA replication.[5] During the G2/M checkpoint process, Ataxia Telangiectasia and Rad3-related (ATR) plays an important role as a sensor of DNA damage in cells.[6] Once DNA damage in cells are sensed by ATR, Checkpoint kinase 1 (Chk1) will be activated. Then Chk1 will inhibit the activity of cell division control protein 25 (Cdc25).[7] The function of Cdc25 in G2/M checkpoint is promoting cells enter M phase by activating the complex of cyclin-dependent kinases (CDK1)/Cyclin B1. Inhibition of Cdc25C will cause cell cycle arrest at G2 phase.[8]

Many kinds of viruses can give rise to cell cycle arrest by activating the mechanisms of checkpoints.[9] Vpr and Vif, important proteins of HIV-1, could induce the cell cycle arrest by interacting with ATR, Cdc25C, CDK1-Cyclin B1 and so on.[10-13] The double strand break (DSB) caused by HIV-1 integration, which is necessary for HIV-1 replication, contributes to the cell cycle arrest and G2/M checkpoint also influence the integration of HIV-1.[14] In addition, many 
reports also showed that the genetic variants of $\mathrm{G} 2 / \mathrm{M}$ checkpoint genes were associated with many kinds of diseases, i.e., the polymorphisms of ATR, Chk1 and CDK1 gene were significantly associated with breast cancer, while the polymorphisms of $C d c 25 C$ gene with hepatocellular carcinoma and so on.[15-17] However, still there is no clear report about the association between the genetic polymorphisms of ATR, Chk1, Cdc25C and CDK1 gene in G2/M checkpoint and susceptibility to HIV-1 infection and AIDS progression in northern Chinese MSM population.

\section{Results}

\subsection{Characteristics of the study subjects}

The basic characteristics and clinical parameters of cases and controls were shown in the Table 1. There was no significant difference between cases and controls for age $(P=0.458)$. The detailed information of HWE test was listed in Table 2. All these tSNPs in controls were in HWE $(P>0.05)$ except Chk1 rs537046 (P=0.049) and CDK1 rs16915503 ( $P=0.029)$, which were excluded from further analysis.

Table 1

Clinical parameters of cases and controls

\begin{tabular}{|c|c|c|c|}
\hline \multirow{2}{*}{$\begin{array}{l}\text { Clinical } \\
\text { Characteristics }\end{array}$} & \multicolumn{2}{|l|}{ Group } & \multirow{2}{*}{$\begin{array}{l}\mathrm{P} \\
\text { value }\end{array}$} \\
\hline & Case $(n=529)$ & Control $(n=529)$ & \\
\hline Age range, years & $16-80$ & $16-75$ & - \\
\hline Mean age $\pm S D$, years & $39.09 \pm 10.57$ & $38.60 \pm 10.97$ & $0.458^{a}$ \\
\hline \multicolumn{4}{|l|}{ Gender n (\%) } \\
\hline Male & 529 (100\%) & 529 (100\%) & - \\
\hline Female & - & - & - \\
\hline \multicolumn{4}{|l|}{ Clinical stages, n (\%) } \\
\hline प & 179 (33.84\%) & - & - \\
\hline ૫ & $144(27.22 \%)$ & - & - \\
\hline ૫ & $144(27.22 \%)$ & - & - \\
\hline ૫ & $62(11.72 \%)$ & - & - \\
\hline \multicolumn{4}{|c|}{$\mathrm{CD}^{+} \mathrm{T}$ cell counts $\left(\right.$ cell $\left./ \mathrm{mm}^{3}\right), \mathrm{n}(\%)$} \\
\hline$\nabla 200$ & $104(19.66 \%)$ & - & - \\
\hline $200-349$ & $124(23.44 \%)$ & - & - \\
\hline $350-500$ & $156(29.49 \%)$ & - & - \\
\hline$\nabla 500$ & $145(27.41 \%)$ & - & - \\
\hline a Student's $t$-test & & & \\
\hline
\end{tabular}


Table 2

The information and allele frequencies of the 42 tSNPs in cases and controls

\begin{tabular}{|c|c|c|c|c|c|c|c|c|}
\hline SNP & Gene & $\begin{array}{l}\text { CHR } \\
\text { a }\end{array}$ & $\begin{array}{l}\text { Risk } \\
\text { Allele }\end{array}$ & Case & Control & $\begin{array}{l}P \\
\text { value }\end{array}$ & OR(95\%Cl) & $\begin{array}{l}\text { P value }{ }^{b} \\
\text { for } \\
\text { HWET c }\end{array}$ \\
\hline rs6780250 & $A T R$ & 3 & $\mathrm{C}$ & $528(0.501)$ & $518(0.490)$ & 0.602 & $\begin{array}{l}1.046(0.882- \\
1.241)\end{array}$ & 0.236 \\
\hline rs145813077 & $A T R$ & 3 & $\mathrm{C}$ & $32(0.030)$ & $31(0.029)$ & 0.892 & $\begin{array}{l}1.035(0.627- \\
1.709)\end{array}$ & 0.488 \\
\hline rs77147770 & $A T R$ & 3 & $\mathrm{~T}$ & 45(0.044) & $33(0.031)$ & 0.146 & $\begin{array}{l}1.402(0.888- \\
2.216)\end{array}$ & 0.457 \\
\hline rs75069062 & ATR & 3 & $\mathrm{~T}$ & $32(0.031)$ & $32(0.030)$ & 0.950 & $\begin{array}{l}1.016(0.618- \\
1.671)\end{array}$ & 0.472 \\
\hline rs200611164 & $A T R$ & 3 & $A$ & $54(0.052)$ & $50(0.048)$ & 0.658 & $\begin{array}{l}1.093(0.737- \\
1.622)\end{array}$ & 0.251 \\
\hline rs34660854 & ATR & 3 & $A$ & $242(0.229)$ & 205(0.194) & 0.049 & $\begin{array}{l}1.234(1.001- \\
1.521)\end{array}$ & 0.249 \\
\hline rs10804682 & ATR & 3 & G & $1016(0.960)$ & $1009(0.954)$ & 0.453 & $\begin{array}{l}1.175(0.771- \\
1.789)\end{array}$ & 0.264 \\
\hline rs73240305 & $A T R$ & 3 & $A$ & $957(0.906)$ & $946(0.894)$ & 0.353 & $\begin{array}{l}1.144(0.861- \\
1.522)\end{array}$ & 0.974 \\
\hline rs75368165 & $A T R$ & 3 & $A$ & $224(0.213)$ & 181(0.173) & 0.020 & $\begin{array}{l}1.296(1.042- \\
1.611)\end{array}$ & 0.101 \\
\hline rs4683425 & $A T R$ & 3 & $A$ & $981(0.956)$ & $963(0.944)$ & 0.212 & $\begin{array}{l}1.290(0.865- \\
1.925)\end{array}$ & 0.238 \\
\hline rs77627941 & ATR & 3 & $A$ & $127(0.122)$ & 106(0.101) & 0.132 & $\begin{array}{l}1.233(0.938- \\
1.621)\end{array}$ & 0.759 \\
\hline rs2227929 & ATR & 3 & G & $433(0.410)$ & $402(0.381)$ & 0.168 & $\begin{array}{l}1.131(0.950- \\
1.346)\end{array}$ & 0.924 \\
\hline rs68065420 & $A T R$ & 3 & $A$ & $385(0.367)$ & $352(0.334)$ & 0.116 & $\begin{array}{l}1.155(0.965- \\
1.381)\end{array}$ & 0.811 \\
\hline rs117312638 & ATR & 3 & $\mathrm{~T}$ & $89(0.084)$ & 86(0.081) & 0.813 & $\begin{array}{l}1.038(0.762- \\
1.415)\end{array}$ & 0.145 \\
\hline rs35514263 & ATR & 3 & $\mathrm{~T}$ & $148(0.141)$ & 145(0.138) & 0.796 & $\begin{array}{l}1.033(0.807- \\
1.323)\end{array}$ & 0.706 \\
\hline rs1057733 & Chk1 & 11 & $\mathrm{~T}$ & $645(0.613)$ & $620(0.587)$ & 0.223 & $\begin{array}{l}1.114(0.936- \\
1.327)\end{array}$ & 0.857 \\
\hline
\end{tabular}

The values in bold indicate statistical significance (P凶0.05);

a Chr: chromosome;

${ }^{b} \mathrm{P}$ value: $\mathrm{P}$ value of HWET in controls;

${ }^{c}$ HWET: Hardy-Weinberg equilibrium test. 


\begin{tabular}{|c|c|c|c|c|c|c|c|c|}
\hline SNP & Gene & $\begin{array}{l}\text { CHR } \\
\text { a }\end{array}$ & $\begin{array}{l}\text { Risk } \\
\text { Allele }\end{array}$ & Case & Control & $\begin{array}{l}P \\
\text { value }\end{array}$ & $\mathrm{OR}(95 \% \mathrm{Cl})$ & $\begin{array}{l}\text { P value }{ }^{b} \\
\text { for } \\
\text { HWET }^{c}\end{array}$ \\
\hline rs558351 & Chk1 & 11 & C & $637(0.603)$ & $620(0.587)$ & 0.451 & $\begin{array}{l}1.069(0.899- \\
1.272)\end{array}$ & 0.857 \\
\hline rs12576279 & Chk1 & 11 & $\mathrm{~T}$ & $927(0.880)$ & $918(0.873)$ & 0.632 & $\begin{array}{l}1.065(0.822- \\
1.381)\end{array}$ & 0.834 \\
\hline rs3731424 & Chk1 & 11 & $\mathrm{~T}$ & $104(0.099)$ & $92(0.087)$ & 0.339 & $\begin{array}{l}1.154(0.8599- \\
1.55)\end{array}$ & 0.590 \\
\hline rs537046 & Chk1 & 11 & A & $811(0.771)$ & 797(0.756) & 0.426 & $\begin{array}{l}1.085(0.887- \\
1.327)\end{array}$ & 0.049 \\
\hline rs10893405 & Chk1 & 11 & A & $865(0.821)$ & $863(0.816)$ & 0.766 & $\begin{array}{l}1.034(0.829- \\
1.290)\end{array}$ & 0.251 \\
\hline rs3731438 & Chk1 & 11 & A & $878(0.831)$ & $862(0.815)$ & 0.315 & $\begin{array}{l}1.122(0.897- \\
1.403)\end{array}$ & 0.363 \\
\hline rs540436 & Chk1 & 11 & $\mathrm{~T}$ & 195(0.186) & $164(0.156)$ & 0.063 & $\begin{array}{l}1.241(0.988- \\
1.558)\end{array}$ & 0.936 \\
\hline rs3731450 & Chk1 & 11 & A & $27(0.026)$ & $17(0.016)$ & 0.133 & $\begin{array}{l}1.594(0.864- \\
2.943)\end{array}$ & 0.706 \\
\hline rs3731466 & Chk1 & 11 & $\mathrm{~T}$ & $111(0.117)$ & 102(0.105) & 0.415 & $\begin{array}{l}1.126(0.847- \\
1.497)\end{array}$ & 0.754 \\
\hline rs75219635 & Chk1 & 11 & C & $41(0.039)$ & $38(0.036)$ & 0.712 & $\begin{array}{l}1.089(0.694- \\
1.707)\end{array}$ & 0.392 \\
\hline rs565435 & Chk1 & 11 & C & $242(0.229)$ & $241(0.228)$ & 0.959 & $\begin{array}{l}1.005(0.821- \\
1.232)\end{array}$ & 0.720 \\
\hline rs74457900 & $C d c 25 C$ & 5 & A & $290(0.284)$ & $285(0.281)$ & 0.893 & $\begin{array}{l}1.013(0.836- \\
1.229)\end{array}$ & 0.267 \\
\hline rs3734166 & Cdc25C & 5 & G & $409(0.389)$ & $396(0.374)$ & 0.493 & $\begin{array}{l}1.063(0.892- \\
1.268)\end{array}$ & 0.984 \\
\hline rs6861656 & $C d c 25 C$ & 5 & T & $773(0.740)$ & $750(0.713)$ & 0.158 & $\begin{array}{l}1.149(0.948- \\
1.392)\end{array}$ & 0.572 \\
\hline rs3756766 & Cdc25C & 5 & A & $163(0.160)$ & $120(0.120)$ & 0.011 & $\begin{array}{l}1.392(1.080- \\
1.794)\end{array}$ & 0.239 \\
\hline rs16915503 & CDK1 & 10 & C & $35(0.033)$ & $32(0.031)$ & 0.801 & $\begin{array}{l}1.065(0.654- \\
1.733)\end{array}$ & 0.029 \\
\hline rs139245206 & $C D K 1$ & 10 & A & $99(0.094)$ & $89(0.084)$ & 0.453 & $\begin{array}{l}1.122(0.831- \\
1.514)\end{array}$ & 0.324 \\
\hline
\end{tabular}

The values in bold indicate statistical significance (P凶0.05);

${ }^{a}$ Chr: chromosome;

b $P$ value: $P$ value of HWET in controls;

${ }^{c}$ HWET: Hardy-Weinberg equilibrium test. 


\begin{tabular}{|c|c|c|c|c|c|c|c|c|}
\hline SNP & Gene & $\begin{array}{l}\text { CHR } \\
\text { a }\end{array}$ & $\begin{array}{l}\text { Risk } \\
\text { Allele }\end{array}$ & Case & Control & $\begin{array}{l}P \\
\text { value }\end{array}$ & $\mathrm{OR}(95 \% \mathrm{Cl})$ & $\begin{array}{l}\text { P value }{ }^{b} \\
\text { for } \\
\text { HWET }^{c}\end{array}$ \\
\hline rs2448343 & CDK1 & 10 & G & $840(0.797)$ & $836(0.792)$ & 0.763 & $\begin{array}{l}1.033(0.836- \\
1.276)\end{array}$ & 0.442 \\
\hline rs3213031 & CDK1 & 10 & G & $90(0.086)$ & $77(0.074)$ & 0.288 & $\begin{array}{l}1.187(0.865- \\
1.630)\end{array}$ & 0.915 \\
\hline rs3213032 & CDK1 & 10 & G & $963(0.912)$ & $942(0.890)$ & 0.097 & $\begin{array}{l}1.275(0.957- \\
1.698)\end{array}$ & 0.052 \\
\hline rs2448345 & CDK1 & 10 & $\mathrm{~T}$ & $927(0.883)$ & $919(0.874)$ & 0.515 & $\begin{array}{l}1.091(0.840- \\
1.417)\end{array}$ & 0.306 \\
\hline rs3213046 & $C D K 1$ & 10 & $\mathrm{~T}$ & $893(0.852)$ & $888(0.843)$ & 0.541 & $\begin{array}{l}1.077(0.849- \\
1.366)\end{array}$ & 0.181 \\
\hline rs2448347 & CDK1 & 10 & A & $759(0.720)$ & $743(0.702)$ & 0.366 & $\begin{array}{l}1.091(0.904- \\
1.317)\end{array}$ & 0.818 \\
\hline rs3213048 & $C D K 1$ & 10 & C & $381(0.365)$ & $367(0.348)$ & 0.405 & $\begin{array}{l}1.079(0.902- \\
1.290)\end{array}$ & 0.669 \\
\hline rs1871445 & CDK1 & 10 & C & $669(0.636)$ & $660(0.629)$ & 0.726 & $\begin{array}{l}1.032(0.864- \\
1.232)\end{array}$ & 0.789 \\
\hline rs3213082 & $C D K 1$ & 10 & C & $1011(0.956)$ & $1007(0.954)$ & 0.827 & $\begin{array}{l}1.047(0.695- \\
1.576)\end{array}$ & 0.396 \\
\hline \multicolumn{9}{|c|}{ The values in bold indicate statistical significance (P凶0.05); } \\
\hline \multicolumn{9}{|c|}{ a Chr: chromosome; } \\
\hline \multicolumn{9}{|c|}{${ }^{b} \mathrm{P}$ value: $\mathrm{P}$ value of $\mathrm{HWET}$ in controls; } \\
\hline
\end{tabular}

\subsection{Allele and genotype associations}

The results showed that allele frequencies of rs34660854-A and rs75368165-A in ATR gene and rs3756766-A in Cdc25C gene were significantly higher in cases than those in controls which suggested that these alleles could increase the susceptibility to HIV-1 infection $(P=0.049, \mathrm{OR}=1.234,95 \% \mathrm{Cl}=0.001-1.521 ; \mathrm{P}=0.020, \mathrm{OR}=1.296,95 \%$ $\mathrm{Cl}=1.042-1.611 ; \mathrm{P}=0.011, \mathrm{OR}=1.392,95 \% \mathrm{Cl}=1.080-1.794$, respectively) (Table 2). Genotype analyses revealed an increased risk for HIV-1 infection with OR value ranging from 1.337 to 1.460 for rs34660854 and rs75368165 in ATR gene, rs540436 in Chk1 gene and rs3756766 in Cdc25C gene under dominant and codominant model $(P<0.05)$. In contrast, a decreased risk for HIV-1 infection was observed of Chk1 rs12576279 under dominant and codominant model $(P=0.048, O R=0.437,95 \% \mathrm{Cl}=0.192-0.991 ; \mathrm{P}=0.013, \mathrm{OR}=0.343,95 \% \mathrm{Cl}=0.147-0.800)$. Moreover, under recessive and codominant model, $C D K 1$ rs 139245206 showed significant differences between cases and controls $(\mathrm{P}$ $=0.020, \mathrm{OR}=5.067,95 \% \mathrm{Cl}=1.286-19.970 ; \mathrm{P}=0.022, \mathrm{OR}=5.011,95 \% \mathrm{Cl}=1.267-19.816)$ (Fig. 1). There was no significant association found between genotype frequency of the other tSNPs and HIV-1infection under different genetic models $(P>0.05)$ (Additional file 1 ).

\subsection{Haplotype analysis}


Strong linkage disequilibrium among tSNPs were observed and thirteen haplotypes in block 1 and block 2 of Chk1 gene and four haplotypes in block 1 of $C d c 25 C$ gene were identified (Additional file 2). A significant difference in haplotype distributions of $C h k 1$ and $C d c 25 C$ gene between cases and controls were found (Table 3 ). The frequencies of H7 (haplotype CCTCGACGC) of Chk1 gene and H4 (haplotype GGT) of Cdc25C gene in block 1 were higher in cases than in controls $(P=0.0054$ and $P=0.0009$, respectively). The association remained significant after correcting for multiple testing using 10,000 permutations with the Haploview program $(P=0.0452$ and $P=0.0017)$. There was no significant difference in frequencies of other haplotypes $(P>0.05)$. 
Table 3

Distribution of haplotypes of Chk1 and Cdc25C gene in cases and controls

\begin{tabular}{|c|c|c|c|c|c|c|c|}
\hline Gene & Block & Haplotypes & Frequencies & $\begin{array}{l}\text { Cases } \\
\text { ratios }\end{array}$ & $\begin{array}{l}\text { Controls } \\
\text { ratios }\end{array}$ & $\begin{array}{l}P \\
\text { value }\end{array}$ & $\begin{array}{l}\text { Permutation } \mathrm{P} \\
\text { value }^{\mathrm{a}}\end{array}$ \\
\hline \multirow[t]{13}{*}{ Chk1 } & $\begin{array}{l}\text { Block } \\
1\end{array}$ & $\begin{array}{l}\text { H1- } \\
\text { TTTCAACGC }\end{array}$ & 0.223 & 0.226 & 0.224 & 0.9350 & 1.0000 \\
\hline & & $\begin{array}{l}\text { H2- } \\
\text { TTTCAGCGC }\end{array}$ & 0.175 & 0.167 & 0.184 & 0.2912 & 0.9771 \\
\hline & & $\begin{array}{l}\text { H3- } \\
\text { TCTCAATGC }\end{array}$ & 0.148 & 0.159 & 0.140 & 0.2069 & 0.9328 \\
\hline & & $\begin{array}{l}\mathrm{H} 4- \\
\text { CCGCAACGC }\end{array}$ & 0.111 & 0.104 & 0.120 & 0.2722 & 0.9701 \\
\hline & & $\begin{array}{l}\text { H5- } \\
\text { CCTCGACGT }\end{array}$ & 0.107 & 0.113 & 0.102 & 0.4050 & 0.9968 \\
\hline & & $\begin{array}{l}\text { H6- } \\
\text { CCTTAACGC }\end{array}$ & 0.091 & 0.097 & 0.086 & 0.4165 & 0.9975 \\
\hline & & $\begin{array}{l}\text { H7- } \\
\text { CCTCGACGC }\end{array}$ & 0.051 & 0.038 & 0.065 & 0.0054 & 0.0452 \\
\hline & & $\begin{array}{l}\text { H8- } \\
\text { CCTCAACGC }\end{array}$ & 0.036 & 0.034 & 0.039 & 0.5776 & 0.9998 \\
\hline & & $\begin{array}{l}\text { H9- } \\
\text { TCTCAATAC }\end{array}$ & 0.020 & 0.025 & 0.016 & 0.1249 & 0.6983 \\
\hline & & $\begin{array}{l}\text { H10- } \\
\text { TCTCGACGC }\end{array}$ & 0.020 & 0.024 & 0.016 & 0.1954 & 0.9210 \\
\hline & $\begin{array}{l}\text { Block } \\
2\end{array}$ & H11-TC & 0.734 & 0.732 & 0.736 & 0.8365 & 1.0000 \\
\hline & & H12-TG & 0.228 & 0.229 & 0.228 & 0.9587 & 1.0000 \\
\hline & & $\mathrm{H} 13-\mathrm{CC}$ & 0.037 & 0.039 & 0.036 & 0.7145 & 1.0000 \\
\hline \multirow[t]{4}{*}{ Cdc25C } & $\begin{array}{l}\text { Block } \\
1\end{array}$ & H1-GAT & 0.344 & 0.334 & 0.354 & 0.3354 & 0.7235 \\
\hline & & H2-AAT & 0.274 & 0.276 & 0.271 & 0.7715 & 0.9959 \\
\hline & & H3-GGC & 0.266 & 0.254 & 0.279 & 0.1901 & 0.4592 \\
\hline & & H4-GGT & 0.105 & 0.128 & 0.083 & 0.0009 & 0.0017 \\
\hline \multicolumn{8}{|c|}{ The values in bold indicate statistical significance $(\mathrm{P} \otimes 0.05)$; } \\
\hline
\end{tabular}

\subsection{Association analysis between SNPs and CD4 ${ }^{+}$T cell counts and clinical phase}

In general, the standard of drug treatment for HIV-1/AIDS was 200 cells $/ \mathrm{mm}^{3}$ of CD4 ${ }^{+} \mathrm{T}$ cell counts. It was selected as the standard to assess the concentrations of $\mathrm{CD} 4^{+} \mathrm{T}$ cells. HIV-1 individuals were classified into two categories 
according to the WHO classification system: pre-AIDS phase (clinical stages I, II, and III) and AIDS phase (clinical stage IV). There was a significant association between the ATR rs75069062 and Chk1 rs10893405 and the clinical stage of AIDS. In detail, rs75069062 showed a significant difference between category A (clinical phase I+ II + III) and category $B$ (clinical IV) $(P=0.026)$. And the frequency of rs10893405 $\mathrm{G}$ allele was higher in category $B$ than that in category $A(P=0.026, O R=1.629,95 \% \mathrm{Cl}=1.051-2.523)$ (Table 4). No significant association was observed between the tSNPs and $\mathrm{CD} 4^{+} \mathrm{T}$ cell counts (Additional file 3 ). 
Table 4

Association between the 40 tSNPs in 4 genes and AIDS clinical stages

\begin{tabular}{|c|c|c|c|c|c|c|c|c|c|}
\hline \multirow{3}{*}{$\begin{array}{l}\text { SNP } \\
\text { rs6780250 }\end{array}$} & \multirow{3}{*}{$\begin{array}{l}\text { Gene } \\
\text { ATR }\end{array}$} & \multirow{2}{*}{\multicolumn{2}{|c|}{ Allele }} & \multicolumn{6}{|c|}{ Clinical stage $^{a}$} \\
\hline & & & & \multicolumn{2}{|c|}{$\bigotimes+\square+\square$} & \multicolumn{2}{|l|}{ प } & \multirow{2}{*}{$\begin{array}{l}\text { P value } \\
0.831\end{array}$} & \multirow{2}{*}{$\begin{array}{l}\text { OR }(95 \% \mathrm{Cl}) \\
0.960(0.660-1.396)\end{array}$} \\
\hline & & $\mathrm{C}$ & $\mathrm{T}$ & 467 & 463 & 61 & 63 & & \\
\hline rs145813077 & $A T R$ & C & $\mathrm{T}$ & 30 & 902 & 2 & 122 & 0.327 & $0.493(0.120-2.029)$ \\
\hline rs77147770 & $A T R$ & $\mathrm{~T}$ & C & 43 & 867 & 2 & 122 & 0.111 & $0.331(0.085-1.290)$ \\
\hline rs75069062 & ATR & $\mathrm{T}$ & C & 32 & 884 & 0 & 122 & 0.026 & - \\
\hline rs200611164 & ATR & A & G & 47 & 869 & 7 & 115 & 0.777 & $1.125(0.497-2.548)$ \\
\hline rs34660854 & ATR & $A$ & G & 216 & 718 & 26 & 98 & 0.591 & $0.882(0.558-1.394)$ \\
\hline rs10804682 & ATR & $\mathrm{G}$ & A & 897 & 37 & 119 & 5 & 0.970 & $0.982(0.378-2.547)$ \\
\hline rs73240305 & ATR & $A$ & G & 845 & 87 & 112 & 12 & 0.902 & $0.961(0.509-1.813)$ \\
\hline rs75368165 & ATR & $A$ & C & 202 & 726 & 22 & 102 & 0.304 & $0.775(0.477-1.259)$ \\
\hline rs4683425 & ATR & $A$ & G & 863 & 39 & 118 & 6 & 0.793 & $0.889(0.369-2.143)$ \\
\hline rs77627941 & $A T R$ & $A$ & G & 114 & 806 & 13 & 109 & 0.582 & $0.843(0.460-1.547)$ \\
\hline rs2227929 & $A T R$ & G & A & 384 & 548 & 49 & 75 & 0.720 & $0.932(0.636-1.367)$ \\
\hline rs68065420 & ATR & $A$ & $\mathrm{C}$ & 341 & 585 & 44 & 80 & 0.771 & $0.944(0.638-1.395)$ \\
\hline rs117312638 & $A T R$ & $\mathrm{~T}$ & $\mathrm{C}$ & 79 & 855 & 10 & 114 & 0.882 & $0.949(0.478-1.886)$ \\
\hline rs35514263 & ATR & $\mathrm{T}$ & $\mathrm{C}$ & 130 & 792 & 18 & 106 & 0.901 & $1.035(0.607-1.763)$ \\
\hline rs1057733 & Chk1 & $\mathrm{T}$ & $\mathrm{C}$ & 570 & 360 & 75 & 47 & 0.969 & $1.008(0.684-1.485)$ \\
\hline rs558351 & Chk1 & C & $\mathrm{T}$ & 558 & 374 & 79 & 45 & 0.412 & $1.177(0.798-1.735)$ \\
\hline rs12576279 & Chk1 & $\mathrm{T}$ & G & 813 & 117 & 114 & 10 & 0.147 & $1.641(0.841-3.202)$ \\
\hline rs3731424 & Chk1 & $\mathrm{T}$ & $\mathrm{C}$ & 91 & 831 & 13 & 111 & 0.830 & $1.069(0.579-1.976)$ \\
\hline rs10893405 & Chk1 & G & A & 158 & 772 & 31 & 93 & 0.029 & $1.629(1.051-2.523)$ \\
\hline rs3731438 & Chk1 & A & G & 772 & 160 & 106 & 18 & 0.459 & $1.220(0.720-2.068)$ \\
\hline rs540436 & Chk1 & $\mathrm{T}$ & C & 171 & 753 & 24 & 100 & 0.820 & $1.057(0.657-1.700)$ \\
\hline rs3731450 & Chk1 & A & G & 22 & 910 & 5 & 119 & 0.268 & $1.738(0.654-4.620)$ \\
\hline rs3731466 & Chk1 & $\mathrm{T}$ & $\mathrm{C}$ & 95 & 741 & 16 & 100 & 0.445 & $1.248(0.707-2.203)$ \\
\hline rs75219635 & Chk1 & $\mathrm{C}$ & $\mathrm{T}$ & 38 & 890 & 3 & 121 & 0.365 & $0.581(0.179-1.883)$ \\
\hline rs565435 & Chk1 & C & $G$ & 719 & 215 & 97 & 27 & 0.756 & $1.074(0.683-1.690)$ \\
\hline
\end{tabular}

The values in bold indicate statistical significance (P凶0.05);

a Clinical stage: Category A, Clinical phase I + II + III; Category B, Clinical phase IV. 


\begin{tabular}{|c|c|c|c|c|c|c|c|c|c|}
\hline \multirow{3}{*}{$\begin{array}{l}\text { SNP } \\
\text { rs74457900 }\end{array}$} & \multirow{3}{*}{$\begin{array}{l}\text { Gene } \\
\text { Cdc25C }\end{array}$} & \multirow{2}{*}{\multicolumn{2}{|c|}{ Allele }} & \multicolumn{6}{|c|}{ Clinical stage $^{a}$} \\
\hline & & & & \multicolumn{2}{|c|}{$\bigotimes+\square+\bigotimes$} & \multicolumn{2}{|l|}{$\square$} & \multirow{2}{*}{$\begin{array}{l}\text { P value } \\
0.935\end{array}$} & \multirow{2}{*}{$\begin{array}{l}\text { OR }(95 \% \mathrm{Cl}) \\
1.018(0.670-1.546)\end{array}$} \\
\hline & & $A$ & G & 255 & 645 & 35 & 87 & & \\
\hline rs3734166 & Cdc25C & $G$ & $A$ & 362 & 568 & 47 & 75 & 0.932 & $0.983(0.667-1.449)$ \\
\hline rs6861656 & Cdc25C & $\mathrm{T}$ & $\mathrm{C}$ & 682 & 238 & 91 & 33 & 0.859 & $0.962(0.629-1.472)$ \\
\hline rs3756766 & Cdc25C & $A$ & $\mathrm{C}$ & 150 & 746 & 13 & 111 & 0.075 & $0.582(0.321-1.055)$ \\
\hline rs139245206 & $C D K 1$ & $A$ & $\mathrm{C}$ & 86 & 848 & 13 & 111 & 0.647 & $1.155(0.624-2.137)$ \\
\hline rs2448343 & CDK1 & G & A & 734 & 196 & 106 & 18 & 0.088 & $1.573(0.935-2.646)$ \\
\hline rs3213031 & $C D K 1$ & G & $A$ & 82 & 838 & 8 & 116 & 0.359 & $0.705(0.334-1.489)$ \\
\hline rs3213032 & $C D K 1$ & G & $A$ & 854 & 78 & 109 & 15 & 0.169 & $0.664(0.370-1.190)$ \\
\hline rs2448345 & CDK1 & $\mathrm{T}$ & C & 816 & 114 & 111 & 9 & 0.127 & $1.723(0.856-3.467)$ \\
\hline rs3213046 & CDK1 & $\mathrm{T}$ & $\mathrm{C}$ & 795 & 131 & 98 & 24 & 0.106 & $0.673(0.416-1.088)$ \\
\hline rs2448347 & $C D K 1$ & $A$ & G & 670 & 260 & 89 & 35 & 0.950 & $0.987(0.651-1.497)$ \\
\hline rs3213048 & CDK1 & C & $\mathrm{T}$ & 339 & 583 & 42 & 80 & 0.614 & $0.903(0.607-1.342)$ \\
\hline rs1871445 & CDK1 & C & $\mathrm{T}$ & 588 & 340 & 81 & 43 & 0.670 & $1.089(0.735-1.614)$ \\
\hline rs3213082 & CDK1 & C & $\mathrm{T}$ & 892 & 42 & 119 & 5 & 0.814 & $1.121(0.435-2.887)$ \\
\hline \multicolumn{10}{|c|}{ The values in bold indicate statistical significance ( $P \otimes 0.05)$; } \\
\hline
\end{tabular}

GMDR was applied to screen the possible gene-gene interaction combinations among 40 tSNPs in 4 genes and the results obtained from GMDR analysis were showed in Table 5. There was only one significant model involving ATR rs68065420, Chk1 rs1057733 and Cdc25C rs6861656 whose cross-validation consistency was 10/10 and test accuracy was 0.5578 . Different combinations of high risk and low risk genotypes of three SNPs in the best model was summarized and an interaction analysis also conducted to explore the association between these combinations and susceptibility to HIV-1 infection (Additional file 4). The individuals carried genotypes of combination 2 and 5 showed a significant decreased in risk for HIV-1 infection $(P=0.047, O R=0.0436,95 \% \mathrm{Cl}=0.192-0.989 ; P=0.004$, $\mathrm{OR}=0.510,95 \% \mathrm{Cl}=0.322-0.809$, respectively). The individuals carried genotypes of combination 14, 18 and 27 showed a significant increased risk for HIV-1 infection $(P=0.0012, O R=1.855,95 \% \mathrm{Cl}=1.146-3.003 ; P=0.008, O R=$ $1.743,95 \% \mathrm{Cl}=1.158-2.623 ; \mathrm{P}=0.015, \mathrm{OR}=3.269,95 \% \mathrm{Cl}=1.255-8.515$, respectively) (Table 6). Additionally, comparing the OR value of these 5 combinations, a trend of risk for HIV- 1 infection progressively increased along with the high risk alleles observed in carrier participants (Fig. 2). 
Table 5

Gene-gene interaction models, as identified by GMDR

\begin{tabular}{|llll|}
\hline Model & Cross-validation consistency & Testing accuracy & P value \\
\hline rs75368165 & $5 / 10$ & 0.4876 & 0.9453 \\
\hline rs34660854 rs540436 & $5 / 10$ & 0.5164 & 0.6230 \\
\hline rs68065420 rs1057733 rs6861656 & $10 / 10$ & 0.5578 & 0.0107 \\
\hline The values in bold indicate the best gene-gene interaction model. & & \\
\hline
\end{tabular}


Interaction between rs68065420, rs1057733 and rs6861656 on HIV-1 risk

\begin{tabular}{|c|c|c|c|c|c|c|c|}
\hline \multirow{2}{*}{$\begin{array}{l}\text { Combination } \\
\text { number }\end{array}$} & \multicolumn{3}{|l|}{ Combination } & \multicolumn{2}{|l|}{ Number } & \multirow{2}{*}{$\begin{array}{l}\mathrm{P} \\
\text { value }\end{array}$} & \multirow[t]{2}{*}{ OR $(95 \% \mathrm{Cl})$} \\
\hline & rs68065420 & rs1057733 & rs6861656 & Cases(n) & Controls(n) & & \\
\hline 1 & $\mathrm{CC}$ & $\mathrm{CC}$ & $\mathrm{CC}$ & 5 & 2 & 0.255 & $\begin{array}{l}2.514(0.514- \\
12.310)\end{array}$ \\
\hline 2 & $\mathrm{CC}$ & $\mathrm{CC}$ & CT & 8 & 18 & 0.047 & $\begin{array}{l}0.436(0.192- \\
0.989)\end{array}$ \\
\hline 3 & $\mathrm{CC}$ & $\mathrm{CC}$ & TT & 20 & 23 & 0.640 & $\begin{array}{l}0.864(0.469- \\
1.593)\end{array}$ \\
\hline 4 & $\mathrm{CC}$ & CT & $\mathrm{CC}$ & 5 & 13 & 0.057 & $\begin{array}{l}0.379(0.139- \\
1.030)\end{array}$ \\
\hline 5 & $\mathrm{CC}$ & $\mathrm{CT}$ & CT & 29 & 54 & 0.004 & $\begin{array}{l}0.510(0.322- \\
0.809)\end{array}$ \\
\hline 6 & $\mathrm{CC}$ & $\mathrm{CT}$ & TT & 61 & 52 & 0.370 & $\begin{array}{l}1.196(0.809- \\
1.768)\end{array}$ \\
\hline 7 & $\mathrm{CC}$ & TT & $\mathrm{CC}$ & 9 & 3 & 0.082 & $\begin{array}{l}3.035(0.870- \\
10.582)\end{array}$ \\
\hline 8 & $\mathrm{CC}$ & TT & CT & 25 & 33 & 0.280 & $\begin{array}{l}0.746(0.438- \\
1.270)\end{array}$ \\
\hline 9 & $\mathrm{CC}$ & TT & TT & 41 & 37 & 0.638 & $\begin{array}{l}1.117(0.704- \\
1.772)\end{array}$ \\
\hline 10 & $A C$ & $\mathrm{CC}$ & $\mathrm{CC}$ & 3 & 1 & 0.316 & $\begin{array}{l}3.011(0.348- \\
26.024)\end{array}$ \\
\hline 11 & $A C$ & $\mathrm{CC}$ & CT & 17 & 13 & 0.459 & $\begin{array}{l}1.318(0.635- \\
2.736)\end{array}$ \\
\hline 12 & $A C$ & $\mathrm{CC}$ & TT & 17 & 18 & 0.864 & $\begin{array}{l}0.943(0.480- \\
1.849)\end{array}$ \\
\hline 13 & $A C$ & $\mathrm{CT}$ & $\mathrm{CC}$ & 11 & 8 & 0.487 & $\begin{array}{l}1.383(0.554- \\
3.453)\end{array}$ \\
\hline 14 & $A C$ & CT & CT & 48 & 27 & 0.012 & $\begin{array}{l}1.855(1.146- \\
3.003)\end{array}$ \\
\hline 15 & $A C$ & CT & TT & 56 & 72 & 0.131 & $\begin{array}{l}0.751(0.518- \\
1.089)\end{array}$ \\
\hline 16 & $A C$ & TT & $\mathrm{CC}$ & 5 & 12 & 0.087 & $\begin{array}{l}0.411(0.149- \\
1.138)\end{array}$ \\
\hline 17 & $A C$ & TT & CT & 23 & 37 & 0.063 & $\begin{array}{l}0.604(0.356- \\
1.027)\end{array}$ \\
\hline 18 & $A C$ & TT & TT & 66 & 40 & 0.008 & $\begin{array}{l}1.743(1.158- \\
2.623)\end{array}$ \\
\hline 19 & $\mathrm{AA}$ & $\mathrm{CC}$ & $\mathrm{CC}$ & 1 & 1 & 1.000 & - \\
\hline
\end{tabular}




\begin{tabular}{|c|c|c|c|c|c|c|c|}
\hline \multirow{2}{*}{$\begin{array}{l}\text { Combination } \\
\text { number }\end{array}$} & \multicolumn{3}{|l|}{ Combination } & \multicolumn{2}{|l|}{ Number } & \multirow{2}{*}{$\begin{array}{l}P \\
\text { value }\end{array}$} & \multirow[t]{2}{*}{ OR $(95 \% \mathrm{Cl})$} \\
\hline & rs68065420 & rs1057733 & rs6861656 & Cases(n) & Controls(n) & & \\
\hline 20 & AA & $\mathrm{CC}$ & CT & 4 & 7 & 0.363 & $\begin{array}{l}0.568(0.168- \\
1.922)\end{array}$ \\
\hline 21 & AA & $\mathrm{CC}$ & TT & 3 & 5 & 0.478 & $\begin{array}{l}0.598(0.144- \\
2.476)\end{array}$ \\
\hline 22 & AA & CT & $\mathrm{CC}$ & 4 & 5 & 0.738 & $\begin{array}{l}0.798(0.214- \\
2.982)\end{array}$ \\
\hline 23 & AA & CT & CT & 14 & 9 & 0.292 & $\begin{array}{l}1.571(0.678- \\
3.636)\end{array}$ \\
\hline 24 & AA & CT & TT & 15 & 16 & 0.855 & $\begin{array}{l}0.936(0.458- \\
1.912)\end{array}$ \\
\hline 25 & AA & TT & $\mathrm{CC}$ & 2 & 1 & 0.563 & $\begin{array}{l}2.004(0.190- \\
21.142)\end{array}$ \\
\hline 26 & AA & TT & CT & 10 & 11 & 0.826 & $\begin{array}{l}0.907(0.382- \\
2.154)\end{array}$ \\
\hline 27 & AA & TT & TT & 16 & 5 & 0.015 & $\begin{array}{l}3.269(1.255- \\
8.515)\end{array}$ \\
\hline
\end{tabular}

\section{Discussion}

It is known that many viruses including HIV-1 induce cell cycle arrest in G2 phase via G2/M checkpoint activation through a variety of mechanisms.[18] HIV-1 is a subtype of HIV, that functions like retrovirus and the integration after reverse transcription is important for its proliferation.[19] The integration of HIV-1 can cause DSB of CD $4^{+}$T cell and activate $\mathrm{G} 2 / \mathrm{M}$ checkpoint that leads to cell cycle arrest.[20,21] This mechanism will also affect the proliferation of HIV-1.[22] Therefore, it is critical to investigate the association between G2/M checkpoint and HIV-1. In this study, 42 tSNPs in ATR, Chk1, Cdc25C and CDK1 gene were genotyped to analysis the association with susceptibility to HIV-1 infection and AIDS progress among MSM population in the northern China.

ATR, as a sensor of DNA damage, contributes to cell cycle arrest, DNA damage repair and stable replication of cells after being activated, which is an important kinase of avoiding apoptosis for cells.[23, 24] A study also found that Vpr-induced structural alteration of DNA can trigger ATR-mediated DNA damage response and contributed to HIV-1 infection.[13] Previous reports showed that rs13091637, which also located in the ATR intronic region and was in strong linkage disequilibrium with rs34660854 in Chinese population of and, was significantly associated with melanoma and breast cancer.[25-27] The results in our study showed that rs34660854-A and rs75368165-A in ATR gene were significantly associated with increased susceptibility to HIV-1 infection. These two SNPs also showed significant differences under codominant and dominant model. It indicated that the rs34660854-A and rs75368165-A in ATR gene were the pathogenic factors for HIV-1 infection. The genotypes carrying risk alleles of these tSNPs were more likely to infect HIV-1 among MSM population in the northern China. By analyzing the effect of the SNPS on AIDS progression, rs75069062 showed a difference between clinical phase I/II/III and clinical phase IV. Although rs34660854, rs75368165 and rs75069062 were all located in the intronic regions, they might be responsible for affecting gene function at transcription level, splicing enhancer or silencer and other mechanisms. 
Chk1 was phosphorylated and activated by ATR after DNA damage had been sensed by ATR at G2/M checkpoint. The genetic mutations of Chk1 gene can cause many kinds of disease such as breast cancers, colorectal cancers, human lymphoid neoplasms and so on.[28-30] However, there are few reports which clearly explain the association between Chk1 and HIV-1 infection. Our results showed that rs12576279 and rs540436 in Chk1 gene were significantly associated with HIV-1 infection risk under codominant and dominant model. The results found that the individuals carrying rs12576279-T were at lower risk for HIV-1 infection. It also indicated that the genotypes including rs540436-T were pathogenic factors for HIV-1 infection. In addition, rs10893405 also showed significant differences between clinical phase I/II/III and IV by analyzing the association between the polymorphisms of Chk1 gene and AIDS progression. It indicated that the rs10893405-G was the risk factor of AIDS progression. Furthermore, a haplotype (H7) and haploid allele of $C h k 1$ gene was significantly associated with HIV-1 infection susceptibility. These three SNPs were all located in the intronic regions and we didn't find any publications about them in NCBI database (https://www.ncbi.nlm.nih.gov). Moreover, the functional studies about these SNPs with HIV-1 infection needed to be carried out.

The protein $\mathrm{Cdc} 25$ is a key inducer for the entry of $\mathrm{M}$ phase and controls the timing of mitosis. It includes three homologues i.e. Cdc25A, Cdc5B and Cdc25C.[31] Cdc25C, which are phosphorylated and inactivated by Chk1, play important roles in the process of $\mathrm{G} 2 / \mathrm{M}$ checkpoint.[32] Many reports showed that Cdc25A and Cdc25B were associated with breast cancers, colorectal cancers, non-small cell lung cancers and so on. But there were few reports about the association between Cdc25C and carcinogenesis.[33] Vpr, which is an important protein of HIV-1, can trigger $\mathrm{G} 2$ arrest by inhibiting the $\mathrm{Cdc} 25 \mathrm{C}$ phosphatase activities. However, no study reported the association between the polymorphisms of $C d c 25 C$ and HIV-1 infection and AIDS progression.[12] Our results showed that rs3756766-A in Cdc25C gene was significantly associated with increased susceptibility to HIV-1 infection. Moreover, this SNP also showed a significant association under codominant and dominant model. It indicated that the rs3456766-A was the pathogenic factor of HIV-1 infection. Hence the genotypes including allele A could increase the cumulative risk of HIV1 infection.

CDK1-Cyclin B1 complex plays an important role in the process of G2/M transition. The activation and nuclear accumulation of this complex are key events for G2/M transition.[34, 35] In the process of G2/M checkpoint, Cdc25C phosphatase activates CDK1 by removing two inhibitory phosphates from Thr14 and Tyr15.[36] A study showed that the Vif of HIV-1 could impair the mitotic entry by interfering with CDK1-Cyclin B1 complex activation causing cell cycle arrest.[10] However, the association of CDK1 polymorphisms and HIV-1infection and AIDS progression remains unclear. The results in our study showed that CDK1 rs139245206 was significantly associated with HIV-1 infection under codominant and recessive model. Although this SNP located in intron regions, it might regulate gene transcription level by binding with transcription factors.

Gene-gene interaction is extremely important, because many genes involve the complex process of G2/M checkpoint regulating cell cycle and the role of a single gene may be finite. Therefore, GMDR software was used to investigate the impact of interaction between ATR, Chk1, Cdc25C and CDK1 gene polymorphisms on HIV-1 infection susceptibility. Our results showed a three-locus model including ATR rs68065420, Chk1 rs1057733 and Cdc25C rs6861656, and participants with rs68065420-AA and rs1057733-TT and rs6861656-TT genotype had the highest HIV-1 infection risk. We also observed a drift of progressively increased risk of HIV-1 infection along with the high risk alleles observed in carrier participants.

\section{Conclusions}


In our study, we investigated the association between genetic polymorphisms of G2/M checkpoint genes and HIV-1 infection and AIDS progression. In conclusion, rs34660854 and rs37568165 in ATR gene, rs12576279 and rs540436 in Chk1 gene, rs3756766 in Cdc25C and rs139245206 in CDK1 gene were associated with susceptibility to HIV-1 infection and rs75069062 in ATR gene and rs10893405 in Chk1 gene were associated with AIDS progression among MSM population in northern Chinese. It revealed that G2/M checkpoint played a crucial role in HIV- 1 infection and AIDS progression. Although, the positive results showed no differences after Bonferroni correction because there were so many SNPs included in this study. However, the results we got were still positive for HIV prevention and should not be ignored due to the false negatives. This finding will provide the theoretical basis and basic data for the prevention of HIV infection and the treatment of AIDS. Moreover, in our further studies, more related researches are needed using larger sample size and different populations to verify our findings.

\section{Methods}

\subsection{Participants}

A total of 529 HIV-1-infected MSM individuals (mean age \pm SD, $39.09 \pm 10.57$ years; age range, 16-80 years) were recruited from the Center for Disease Control and Prevention (CDC) of Heilongjiang Province. Meanwhile, 529 unrelated healthy male individuals age-matched (mean age \pm SD, $38.60 \pm 10.97$ years; age range, $16-75$ years) to the HIV-1 patients were randomly selected from the Second Affiliated Hospital of Harbin Medical University. It is noticed that the proportion of MSM population in HIV-1 infected patients has gradually increased in recent years. Males were selected as the subjects for our investigation. The protocols used in this study were evaluated and approved by the Ethics Committee of Harbin Medical University (No.: HMUIRB20180019) and written informed consent was obtained from all participants.

\section{2 tSNPs selecting}

42 tSNPs of G2/M checkpoint genes were selected to investigate the associations with HIV-1 infection and AIDS progression (Table 2). All tSNPs were selected based on linkage disequilibrium (LD) with Han Chinese in Beijing (CHB) as a reference population $\left(r^{2}>0.03\right)$ by Haploview software (version 4.2$)$.

\subsection{DNA extraction and genotyping}

The DNA of every participant was extracted from peripheral blood of every individual using the QIAamp blood kit (Qiagen, Hilden, Germany). And the genotyping of these 42 tSNPs was carried out utilizing the SNPscan Kit (Genesky Biotechnologies Inc., Shanghai, China). 53 participants (5\% of all participants) were selected randomly whose 42 tSNPs were double-genotyped in order to ensure the accuracy of the results and the accuracy rate was $100 \%$.

\subsection{Statistical analysis}

The difference of age distribution between case and control groups was compared by Student's $t$-test. HardyWeinberg equilibrium (HWE) in the controls was checked by Chi-square test. The associations of these 42 tSNPs with susceptibility to HIV-1 infection and AIDS progression were determined using Chi-square test, and the effect size was expressed as odds ratio (OR) with $95 \%$ confidence interval $(95 \% \mathrm{Cl})$. The statistical analyses were performed by SPSS v. 22.0 statistical software (IBM-SPSS, Inc., Chicago, USA). Haploview v4.2 was used to analyze the linkage disequilibrium (LD) and haplotype, and 10,000 permutations were run to compute the p-values. $P<0.05$ was considered statistically significant. Generalized Multifactor Dimensionality Reduction (GMDR) v 0.9 (http://www.ssg.uab.edu/gmdr/) was utilized to explore the possible gene-gene interaction. 


\section{Abbreviations}

AIDS: Acquired immune deficiency syndrome; ATR:Ataxia Telangiectasia and Rad3-related kinase; Cdc25C:Cell division control protein 25 homolog C; CDK1:Cyclin-dependent kinase 1; Chk1:Checkpoint kinase 1; DSB:Double strand break; GMDR:Generalized multifactor dimensionality reduction; HIV:Human immunodeficiency virus; HWE:Hardy-Weinberg equilibrium; MSM:Men who have sex with men; OR:Odd ratio; SNP:Single nucleotide polymorphism.

\section{Declarations}

\subsection{Ethics approval and consent to participate:}

The protocols used in this study were evaluated and approved by the Ethics Committee of Harbin Medical University (No.: HMUIRB20180019) and written informed consent was obtained from all participants.

\subsection{Consent for publication:}

Not applicable.

\subsection{Availability of data and materials:}

The datasets used and/or analysed during the current study are available from the corresponding author on reasonable request.

\subsection{Competing interests:}

The authors declare that they have no competing interests.

\subsection{Funding:}

This work was funded by the Program for the Postdoctoral Foundation of Heilongjiang Province (LRB08-340) of Yuandong Qiao and Wu lien-the Youth Science Foundation of Harbin Medical University (WLD-QN1405) of Lidan Xu.

\subsection{Authors' contributions:}

Conceptualization: Yuandong Qiao; Data curation: Jiawei Wu, Kaili Wang, Lidan Xu, Bangquan Liu and Yuanting Hu; Funding acquisition: Yuandong Qiao; Investigation: Jiawei Wu, Xuelong Zhang, Kaili Wang and Wenjing Sun; Methodology: Jiawei Wu, Xueyuan Jia, Haiming Sun, Jie Wu, Yun Huang, Wei Ji; Software: Jiawei Wu and Xuelong Zhang; Supervision: Yuandong Qiao; Validation־Yuandong Qiao; Visualization: Jiawei Wu; Writing original draft: Jiawei Wu and Xuelong Zhang; Writing review\& editing: Komal Saleem, Songbin Fu and Yuandong Qiao. All authors read and approved the final manuscript.

\subsection{Acknowledgements:}

We gratefully acknowledge the numerous sample donors for making this work possible. 


\section{References}

1. Whiteside A, Wilson D. Health and AIDS in 2019 and beyond. Afr J AIDS Res. 2018;17:iii-v.

2. Giacinti C, Giordano A. RB and cell cycle progression. Oncogene. 2006;25:5220-7.

3. Wiman KG, Zhivotovsky B. Understanding cell cycle and cell death regulation provides novel weapons against human diseases. J Intern Med. 2017;281:483-95.

4. Barnum KJ, O'Connell MJ. Cell cycle regulation by checkpoints. Methods Mol Biol. 2014;1170:29-40.

5. Luo Q, Guo H, Kuang P, Cui H, Deng H, Liu H, Lu Y, Wei Q, Chen L, Fang J, et al. Sodium Fluoride Arrests Renal G2/M Phase Cell-Cycle Progression by Activating ATM-Chk2-P53/Cdc25C Signaling Pathway in Mice. Cell Physiol Biochem. 2018;51:2421-33.

6. Brown EJ, Baltimore D. Essential and dispensable roles of ATR in cell cycle arrest and genome maintenance. Genes Dev. 2003;17:615-28.

7. Koniaras K, Cuddihy AR, Christopoulos H, Hogg A, O'Connell MJ. Inhibition of Chk1-dependent G2 DNA damage checkpoint radiosensitizes p53 mutant human cells. Oncogene. 2001;20:7453-63.

8. Nilsson I, Hoffmann I. Cell cycle regulation by the Cdc25 phosphatase family. Prog Cell Cycle Res. 2000;4:10714.

9. Zhao RY, Elder RT. Viral infections and cell cycle G2/M regulation. Cell Res. 2005;15:143-9.

10. Sakai K, Barnitz RA, Chaigne-Delalande B, Bidere N, Lenardo MJ. Human immunodeficiency virus type 1 Vif causes dysfunction of Cdk1 and CyclinB1: implications for cell cycle arrest. Virol J. 2011;8:219.

11. He J, Choe S, Walker R, Di Marzio P, Morgan DO, Landau NR. Human immunodeficiency virus type 1 viral protein $\mathrm{R}(\mathrm{Vpr})$ arrests cells in the G2 phase of the cell cycle by inhibiting p34cdc2 activity. J Virol. 1995;69:6705-11.

12. Goh WC, Manel N, Emerman M. The human immunodeficiency virus Vpr protein binds Cdc25C: implications for G2 arrest. Virology. 2004;318:337-49.

13. lijima K, Kobayashi J, Ishizaka Y. Structural alteration of DNA induced by viral protein R of HIV-1 triggers the DNA damage response. Retrovirology. 2018;15:8.

14. Izumi T, lo K, Matsui M, Shirakawa K, Shinohara M, Nagai Y, Kawahara M, Kobayashi M, Kondoh H, Misawa N, et al. HIV-1 viral infectivity factor interacts with TP53 to induce G2 cell cycle arrest and positively regulate viral replication. Proc Natl Acad Sci U S A. 2010;107:20798-803.

15. Li Y, Chen YL, Xie YT, Zheng LY, Han JY, Wang H, Tian XX, Fang WG. Association study of germline variants in CCNB1 and CDK1 with breast cancer susceptibility, progression, and survival among Chinese Han women. PLoS One. 2013;8:e84489.

16. Lin WY, Brock IW, Connley D, Cramp H, Tucker R, Slate J, Reed MW, Balasubramanian SP, Cannon-Albright LA, Camp NJ, Cox A. Associations of ATR and CHEK1 single nucleotide polymorphisms with breast cancer. PLoS One. 2013;8:e68578.

17. Nan YL, Hu YL, Liu ZK, Duan FF, Xu Y, Li S, Li T, Chen DF, Zeng XY. Relationships between cell cycle pathway gene polymorphisms and risk of hepatocellular carcinoma. World J Gastroenterol. 2016;22:5558-67.

18. Ariumi Y, Turelli P, Masutani M, Trono D. DNA damage sensors ATM, ATR, DNA-PKcs, and PARP-1 are dispensable for human immunodeficiency virus type 1 integration. J Virol. 2005;79:2973-8.

19. Hemelaar J. The origin and diversity of the HIV-1 pandemic. Trends Mol Med. 2012;18:182-92.

20. Elder RT, Yu M, Chen M, Zhu X, Yanagida M, Zhao Y. HIV-1 Vpr induces cell cycle G2 arrest in fission yeast (Schizosaccharomyces pombe) through a pathway involving regulatory and catalytic subunits of PP2A and acting on both Wee1 and Cdc25. Virology. 2001;287:359-70. 
21. DeHart JL, Bosque A, Harris RS, Planelles V. Human immunodeficiency virus type 1 Vif induces cell cycle delay via recruitment of the same E3 ubiquitin ligase complex that targets APOBEC3 proteins for degradation. J Virol. 2008;82:9265-72.

22. Lawn SD, Butera ST, Folks TM. Contribution of immune activation to the pathogenesis and transmission of human immunodeficiency virus type 1 infection. Clin Microbiol Rev. 2001;14:753-77. table of contents.

23. Cimprich KA, Cortez D. ATR: an essential regulator of genome integrity. Nat Rev Mol Cell Biol. 2008;9:616-27.

24. Xu B, Kim ST, Lim DS, Kastan MB. Two molecularly distinct G(2)/M checkpoints are induced by ionizing irradiation. Mol Cell Biol. 2002;22:1049-59.

25. Zhang M, Qureshi AA, Guo Q, Han J. Genetic variation in DNA repair pathway genes and melanoma risk. DNA Repair. 2011;10:111-6.

26. Wang K, Ye Y, Xu Z, Zhang X, Hou Z, Cui Y, Song Y. Interaction between BRCA1/BRCA2 and ATM/ATR associate with breast cancer susceptibility in a Chinese Han population. Cancer Genet Cytogenet. 2010;200:40-6.

27. Durocher F, Labrie Y, Soucy P, Sinilnikova O, Labuda D, Bessette P, Chiquette J, Laframboise R, Lepine J, Lesperance $B$, et al. Mutation analysis and characterization of ATR sequence variants in breast cancer cases from high-risk French Canadian breast/ovarian cancer families. BMC Cancer. 2006;6:230.

28. Tort F, Hernandez S, Bea S, Camacho E, Fernandez V, Esteller M, Fraga MF, Burek C, Rosenwald A, Hernandez L, Campo E. Checkpoint kinase 1 (CHK1) protein and mRNA expression is downregulated in aggressive variants of human lymphoid neoplasms. Leukemia. 2005;19:112-7.

29. Bertoni F, Codegoni AM, Furlan D, Tibiletti MG, Capella C, Broggini M. CHK1 frameshift mutations in genetically unstable colorectal and endometrial cancers. Genes Chromosomes Cancer. 1999;26:176-80.

30. Solyom S, Pylkas K, Winqvist R. Screening for large genomic rearrangements of the BRIP1 and CHK1 genes in Finnish breast cancer families. Fam Cancer. 2010;9:537-40.

31. Jinno S, Suto K, Nagata A, Igarashi M, Kanaoka Y, Nojima H, Okayama H. Cdc25A is a novel phosphatase functioning early in the cell cycle. Embo j. 1994;13:1549-56.

32. Zhou BB, Elledge SJ. The DNA damage response: putting checkpoints in perspective. Nature. 2000;408:433-9.

33. Kristjansdottir K, Rudolph J. Cdc25 phosphatases and cancer. Chem Biol. 2004;11:1043-51.

34. Doree M, Galas S. The cyclin-dependent protein kinases and the control of cell division. Faseb j. 1994;8:1114-21.

35. Masui Y. Towards understanding the control of the division cycle in animal cells. Biochem Cell Biol. 1992;70:920-45.

36. Dunphy WG, Kumagai A. The cdc25 protein contains an intrinsic phosphatase activity. Cell. 1991;67:189-96.

\section{Figures}




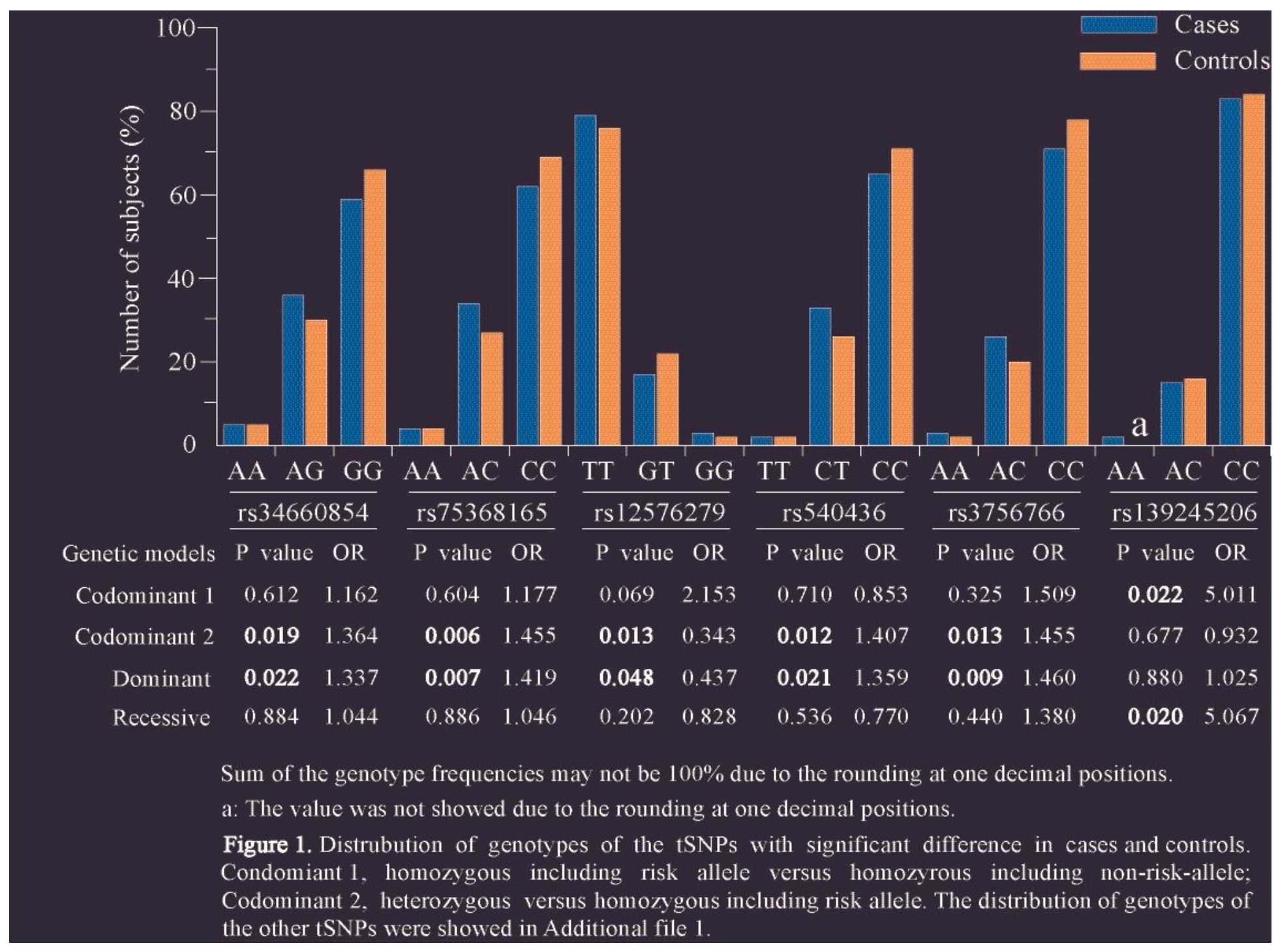

\section{Figure 1}

Distrubution of genotypes of the tSNPs with significant difference in cases and controls. Condomiant 1, homozygous including risk allele versus homozyrous including non-risk-allele; Codominant 2 , heterozygous versus homozygous including risk allele. The distribution of genotypes of the other tSNPs were showed in Additional file 1. 


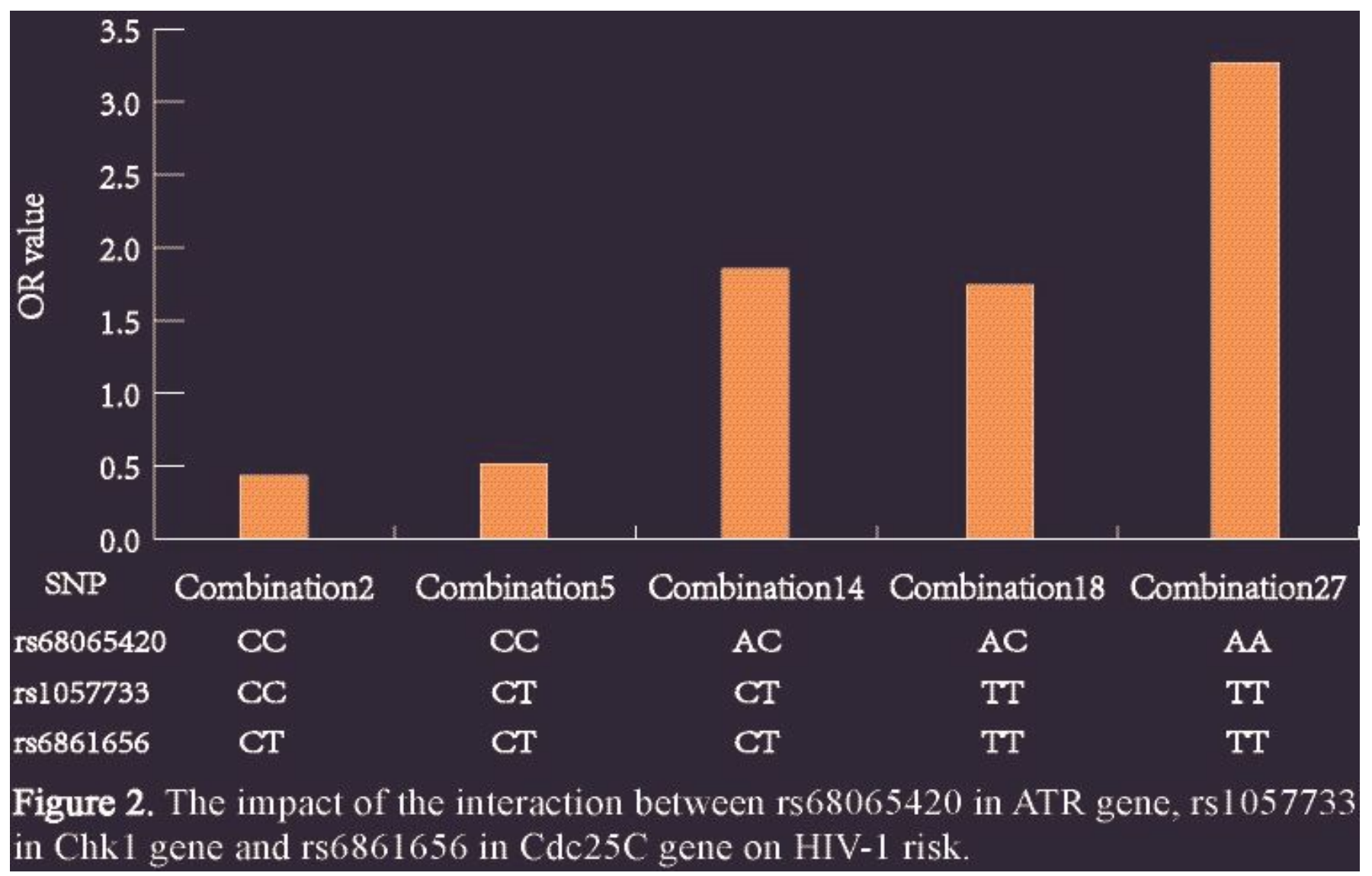

\section{Figure 2}

The impact of the interaction between rs68065420 in ATR gene, rs1057733 in Chkl gene and rs6861656 in Cdc25C gene on HIV-1 risk.

\section{Supplementary Files}

This is a list of supplementary files associated with this preprint. Click to download.

- Additionalfile4.eps

- Additionalfile3.docx

- Additionalfile2.eps

- Additionalfile1.docx 